\title{
An ontology enabled internet of things framework in intelligent agriculture for preventing post-harvest losses
}

\author{
P. Sanjeevi ${ }^{1}$ (D) B. Siva Kumar ${ }^{2}$. S. Prasanna ${ }^{3} \cdot$ J. Maruthupandi $^{4} \cdot$ R. Manikandan ${ }^{5} \cdot$ A. Baseera $^{6}$
}

Received: 29 March 2020 / Accepted: 24 July 2020 / Published online: 11 August 2020

(c) The Author(s) 2020

\begin{abstract}
Constituting the agriculture solid substance manufacture, the post-harvest sector processing schema is direct to preventing reduce the losses in intelligent agriculture. Many processing schemata will be preventing post-harvest losses on the agriculture solid substance manufacture, especially sekai-ichi apple is the regularly used fruit also used to make active in human-related activities of the sensory and control function consisting of an agricultural industry. Sekai-ichi apple is being a definite number of diseases induce, but it is to the highest degree of wastage involving in the Post-Harvest process. Especially sekai-ichi apple count loss is an unsafe many time because it not critically post-harvest. Regardless of consideration, the existing hierarchical model specified post-harvest losses prevention research has deficiencies to precise and quick detection of wastage for ensuring healthy separation of agriculture surroundings. This paper suggests a "Hierarchical Model within Ontology Enabled IoT" for distinguishable healthy separation of sekai-ichi apple by using Boosted Continuous Non-spatial whole Attribute Extraction (BCNAE). Sekai-ichi apple count loss is always safe on critically post-harvest. Proposed PostHarvest hierarchical model specified post-harvest losses prevention and deficiencies to precise and quick detection of wastage for ensuring healthy separation of agriculture surroundings. In these suggestions, the separation cognitive operation takes the three levels of processing schemes such as lower level, middle level, and higher level. Firstly, the lower level is express agreements with the dynamic functioning for maintaining the definite number of manual induces. This lower level showing an absorption with the activity of manual separation by the human reliability determination. Secondly, the middle level is an express arrangement with the dynamic functioning for reducing the overfitting and accommodate to fitting the right shape deliberation. Middle level is establishing being generalized by concentrating the time-varying features in the occurrence of a change for the worse identification. Finally, the upper level is express for features refining with the help of the function of sekai-ichi apple image segmentation connection. This interpretability process helps to make the proven position of a prominent classification in a particular fruit on the agriculture solid substance. These three processing flow constructs the ontology structure with manually collected sekai-ichi apple images from a 3D sensor. The observational consequences express that the proposed BCNAE framework recognizes a detection performance carrying out with an optimized—separation ratio for time-variant of the separation process.
\end{abstract}

Keywords Hierarchical model $\cdot$ Ontology $\cdot$ IoT $\cdot$ Intelligent agriculture $\cdot$ Post-harvest loss $\cdot$ Attribute extraction $\cdot 3 \mathrm{D}$ sensor $\cdot$ Smallest $\mathrm{S}$-vertex $\cdot$ Separation $\cdot$ Spatial image

P. Sanjeevi

gpsanjeevi@jiangnan.edu.cn

1 Key Laboratory of Advanced Process Control for Light Industry, Ministry of Education, Jiangnan University, Wuxi 214122, China

2 Department of Computer Science and Engineering, SRM Institute of Science and Technology, Chennai, India

3 School of Information Technology and Engineering, VIT, Vellore, India
4 Department of Information Technology, Mepco Schlenk Engineering College, Sivakasi, India

5 Department of Computer Science and Engineering, Karpagam Institute of Technology, Coimbatore, India

6 School of Computing Science and Engineering, VIT Bhopal University, Bhopal, India 


\section{Introduction}

From the consideration of constituting the complete agriculture industry and their solid substance manufacture processing in post-harvest sector services is the most precious compositions is the direction preventing reduce the losses in intelligent agriculture [1-16]. From the processing schemata, will be preventing post-harvest losses on the agriculture solid substance (dekopon, densuke watermelon, pineapple, ruby roman grapes, sekai-ichi apple, strawberries arnaud, yubari king melons, and bael or wood apple) manufacture, especially sekai-ichi apple is the regularly used fruit also used to make active in human-related activities of the sensory and control function consisting of an agricultural industry [17, 18]. Sekai-ichi apple is being a definite number of diseases induce, but it is to the highest degree of wastage involving in the Post-Harvest process [1]. Especially sekai-ichi apple count loss is an unsafe many time because it not critically post-harvest [19]. This paper suggests a "Hierarchical Model within Ontology Enabled IoT" for distinguishable healthy separation of sekai-ichi apple [20, 21].

An idea that is suggested above, the separation manual with automated procedure takes being three levels [22] of processing schemes such as lower level, middle level, and higher level. First of all, the lower level is an agreement with the dynamic functioning for maintaining the distinct number of transmission that is operated manually with human hands and a viewing separation by the human being as labor [2, 23]. This lower level establishing a concentration with the activeness by hand separation by the human reliability finding. In the second place, the middle level is conveying organization with the dynamic functioning for reducing the overfitting and accommodate to fitting the right shape deliberation by location.

Middle level establishing generalizable (reduced to a time-variant of detection process) by concentrating the time-varying features in the occurrence of a change for the worse identification [24]. After a specified (that would take by the middle level) period or an especially fewer delay, the upper level is express for features refining with the help of the function of sekai-ichi apple image segmentation [25, 26]. The relation between affected and unaffected sekai-ichi apple (as in the case of one causing the other or sharing features with it) is finalized. This interpretability operation assists to establish the proven perspective of an outstanding categorization in a specific fruit on the agriculture solid substance $[27,28]$. These three treating flow abstract general idea inferred the ontology structure with manually gathered sekai-ichi apple image from the 3D sensor [29, 30].

To maintain the above consideration in the practical verification, this research article to consider and examine
"Ontology-based Separation Cognitive Segmentation" by three process schema [23, 31]. The "Boosted Continuous Non-spatial whole Attribute Extraction (BCNAE)" for extracting the Sekai-ichi apple attributes such as area (location), compactness (the consistency of a compact solid substances), moments (particular spot identification in a time unit), entropy (a numerical measure of the uncertainty of an outcome). Secondly, this research concentrate on Rhombus based recognition and Isolation Guarantee Spotting (RIGS). This rhombus also called the equilateral quadrilateral. In our research concentrate, the tetragon based rhombus was formed [3, 32].

Finally, the ontology level qualities and performance checking was performed for confirmation affected and unaffected sekai-ichi apple separation in the post-harvest [33, 34]. The Boosted Continuous Non-spatial whole Attribute Extraction (BCNAE) is characterized by the following delimiter such as a non-spatial of an image, real-time spotting of a Sekai-ichi apple separation without loss of any authenticity. This proposed approach has been applied to an ontology framework to the spotting of the exact confirmation affected and unaffected sekai-ichi apple separation concerning sekai-ichi apple attributes of the post-harvest extended spatial location of intelligent agriculture [3, 25, 35]. Boosted continuous non-spatial whole attribute extraction (BCNAE) apply thoroughly rhombus based recognition and isolation guarantee spotting (RIGS).

This RIGS accomplishes the separation affected and unaffected sekai-ichi apple attributes with aid of ontology framework [36]. This might be segregate with higher reliability and real-time spotting sekai-ichi apple attributes on postharvest [34, 35]. Furthermore, the recognition and isolation guarantee vital factor for making the ontology. This helps to make better the recognition result of the ontology framework [29, 37]. The "recognition and isolation guarantee" works based on the BCNAE with the original input image from the 3D sensor [20, 23, 31]. This proposed rhombus based recognition and isolation guarantee spotting method is established and also discrimination between recognition and isolation is distinct from existing spot identification methods, the first recognition and isolation are treated the Origin Input Image(a) samples of the 3D sensor image [30, 31].

The 3D sensor image comprises identifying the micro affected spot on a sekai-ichi apple with a continuous scanned image. In that consideration, this influenced distinguishes are named concerning recognition and isolation [33, 38, 39]. Finally, the optimized hierarchical model within ontology enabled IoT in intelligent agriculture for preventing postharvest losses is respond in a quick, clever humorous way of separation of affected and unaffected sekai-ichi apple based on the Smallest S-vertex tetragon based recognition and isolation guarantee spotting algorithm [40-42]. It requires the 
collection of all S-vertex tetragon diagonals. The exact point located concerning the affected spot on the sekai-ichi apple image region is estimated by make work for a particular IoT based Intelligent Agriculture. The experimental moments carry that the suggested model distinguishes a sensing functioning with an optimized-separation ratio is higher timevariant of the separation process $[43,44]$.

The rest of the paper is organized as follows. In "Related review" section discussed related works and are reviewed based on the $3 \mathrm{~d}$ sensor image, apple fruit separation, attributes of continuous non-spatial image, hierarchical model, intelligent agriculture, IoT, ontology, optimized and preventing post-harvest losses [38, 41, 45], recognition and isolation, tetragon based rhombus verification and attribute extraction. "Boosted continuous non-spatial whole attribute extraction (BCNAE)" presents the proposed boosted continuous non-spatial whole attribute extraction (BCNAE) system and its techniques related to the rhombus based recognition and isolation guarantee spotting (RIGS). Also this research article example performance measure discussion is shown in the same Section. "Conclusion" concludes the research work with reference $[20,23,28,46]$.

\section{Related review}

Kamilaris et al. (2019) proposed the Agri-IoT for developing smart farming. This was very much useful to improve the ideologies against the smart agricultural [47]. In the large-scale IoT platforms, their aimed the Agri-IoT helps to accumulate, cognitive operation and examine data for smart solutions in the agri-food industry. They acquired the food supply chain with IoT frameworks. This helped to make smart farming with the context of Agri-IoT [48]. They employ the heterogeneous sensor for data streams in realtime. Their sensing/automation systems work based on event detection, sensors interoperability, information sources, operations, processes, and increased automation services with less effort by employing internet standards. Finally, they used components like the dashboard, data aggregation, data federation, data wrapper, device manager, discovery module, event detection, external agent, knowledgebase, mobile apps and real-time adaptive reasoning for forming the Agri-IoT. This research helps us to make the Agri-IoT with real-time sensing apple fruit for decision making concerning the operations, processes and increased automation. This process allows us to create the real-time stream processing for analysis and reasoning in the domain of agriculture concerning the sensors, capturing camera, manual farms, etc.), this motivates us to achieve separation between sensors and entities based on IoT.

Lutfy et al. (2015) modelled the conveyor-belt grain dryer for technical separation on any object named as a sigmoid-based NARX model [49]. They used the postharvest techniques for the grain drying process. These techniques are commonly having a lower complexity and linearity for accurate modeling results. Their conveyor-belt grain dryer has the nonlinear modeling technique with the sigmoid network. This sigmoid network helps us to make the nonlinearity separation with the nonlinear semi-automatic model. Their system applied to the paddy grains for separation of dry paddy and wet paddy. This research motivates us to create the semi-automatic machine for the intelligent agricultural with IoT. The proposed modes of operation are compared with the manual operation with the proposed sigmoid-based NARX model. The sigmoid-based NARX model returns the achieved lower root mean squared error (RMSE). It motivates them to research for making the manual separation with Intelligent Agricultural with IoT semi-automatic machine create the lowest mean squared error for the separation process.

Lu et al. (2019) discussed the IoT-Enabled Cross-Field. They used the smart and ambient devices for recurring the unidirectional and noncustomizable, properties on the Market and Discover (MnD). They use the Match and Recommend (MnR) for enhance privacy [50]. MnR applies to the end system, the system returns the quicker and more efficient service against the separation of unwanted advertising information with the right information. Moreover, the bidirectionally interactive between the two processes are engaged by using the proposed Meet and Interact $(\mathrm{MnI})$ module. This study helps us to make the cross-field utilization of users input data by location-based service provisioning with IoT application. This research motivates us to make the system with the following modules like user data collection, notifications, virtual separation, and service recommendations. It also provides ideas on negative useless invasive identification. Finally, comparison with other matching accuracy and efficiency in matching speed. This system used for us to make the realizing cross-filed on the IoT-enabled reconfigurable service provisioning with a user-centered design with user privacy.

Lan et al. (2019) proposed the IoT unified access platform. Their research concentrates on the sensing devices for accurate location finding [44]. IoT unified access platform can have used for efficient privacy protection against the sensitive data were applied to the object with the help of the proposed IoT access platform with the network edge. They also proposed the ontology-based resource model of IoT. It will help to provide a consistent view of heterogeneous sensing devices for IoT. This system motivates us to make the unified access, control, and management of IoT devices over the intelligence levels separation process. Their system helps us to make our research architecture of IoT with a universal access platform against sensitive data. Also, support for sharing and

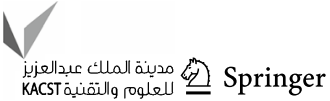


reuse of IoT. Finally, their smart city road manhole cover monitoring system (SCRMCMS) implementation with a Three-layer computing model structure of the "sensing device-IoT access platform-IoT application motivates us to create the Boosted Continuous Non-spatial whole attribute extraction (BCNAE) machine architecture for separation of sekai-ichi apple based on the access platform. The performance evaluation is also used to verify the effectiveness of the access solution.

Hamidi et al. (2018) proposed the internet of things and biosensors for the health rehabilitation of individuals [51]. They proposed an ontology-based automatic design for intelligent rehabilitation systems with the internet of things (IoT). This research helps to provide fast and effective rehabilitation for different patients with the help of IoT with ontology. This helps us to make an effective way of separations from a large number of objects. IoTbased rehabilitation system works for Rehabilitation of the lower limbs. This motivates us to execute the segmentation to the affected and unaffected nodes. Although, the effective rehabilitation strategy was concluded by ontology-based IoT. This helps us to create the ontologybased IoT system against the non-spatial whole Attribute Extraction. This study uses three biosensors. First sensor for blood, the second sensor for saliva and a third sensor for breathing tests. This survey helps us to construct the three 3D sensors. First sensor for harvest finding, a second sensor for confirmation and a third sensor for separation tests. Fisher-Pry model is based on patent and bibliometric analysis used for intellectual property analysis. This study supports us to make the three distinguished "Divisions" on the semi-automatic result image event for the functional behavior structure. Three-level automatic design methodology motivates us to make the human and machine-to-machine interconnection technology with a separation framework with the idea of the IoT.

Guzman-Miranda et al. (2009) proposed the fruit inspection and classification in the post-harvest process with respect to the rapid prototyping and development model [52]. They concentrate the visual inspection for classification on fruit quality. The highly specific problem was resolved on the classification, their system might overcome the accurate design and lower costs with reconfigurable hardware. The modularization and standardized operation were applied for every level of process that can increase the processing quality of the products. They used the color factor for separation. The maximum uniformity with minimum product loss was achieved by the proposed post-harvest process. Speed and temporal limitations are verified on the classification of olives. The cost of classification tasks automated with the proposed system. This research platform motivates us to accurate inspection and separation that can be adapted proposed solutions.

\section{Boosted continuous non-spatial whole attribute extraction (BCNAE)}

The boosted continuous non-spatial whole attribute extraction (BCNAE) is described by the following flow diagram. BCNAE exploit delimiter quantifier such as a Non-spatial of an image, real-time spotting of a Sekai-ichi apple separation without loss of any authenticity. This proposed approach has been concentrating the above two delimiters by improving the authentication against intelligent agriculture [3]. The non-Spatial of an image cannot be contingent upon the specific state of connectedness between the non-spatial region close around the affected and unaffected separation amongst the values; here the non-spatial of an image can be purely close on the unaffected separation. Whereas the spatial of an image cannot be purely close to the affected separation. Hence, a more similar result must suitable for the non-spatial of an image. For this result, the unaffected separation took higher recognition and Isolation observational. Henceforth, the affected separation took the opposite number with lower recognition and isolation observational rates. This acceleration might be continuing until the final non-spatial image were refined. In the post-harvest [53], the above said continuous nonspatial processing helps to boost up the separation process on the affected and unaffected sekai-ichi apple fruit. This research might apply a boosted continuous non-spatial image to the ontology framework for spotting the exact confirmation. The resulted affected and unaffected sekaiichi apple is identified with the attributes of post-harvest [46, 54]. Boosted continuous non-spatial whole attribute extraction (BCNAE) apply thoroughly rhombus based recognition and isolation guarantee spotting (RIGS). As per the Oluwo et al. (2015), they used the optimal fuzzy logic controller (FLC), root mean square error (RMSE) and product mean temperature difference (PMTD), membership functions (MFs). This makes the system is very composite. And also Purandare et al. (2016) also used notification-suggestion system; prediction-analysis and suggestions, various statistical and probabilistic techniques, classification and regression are used. This makes the long procedural aspect to post-harvest. This makes the huge complexity on the post-harvesting. Likewise Sattar et al. (2019) also specifies the IoT based intelligent wound assessment system, entropy and information gain, decision tree, categorization, ID3 algorithm for decision tree for monitoring system. This also makes the major complexity on the monitoring system. Perhaps our research article makes the simple and secure system against the post-harvest, monitoring and storage system.

Figure 1 describes the proposed boosted continuous non-spatial whole attribute extraction (BCNAE) with the 


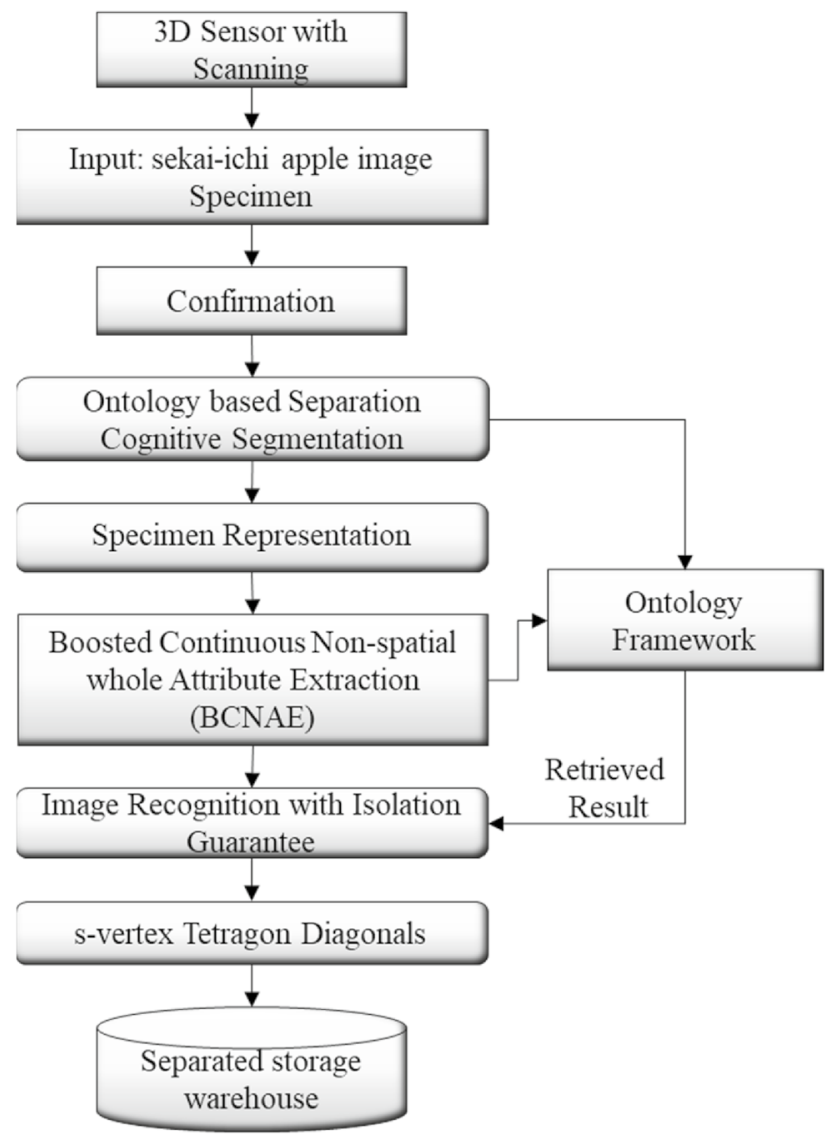

Fig. 1 Boosted continuous non-spatial whole attribute extraction (BCNAE) with RIGS

RIGS scheme with the concept proficiencies used in the sekai-ichi apple fruit separation as well as sekai-ichi apple attributes verification by the ontology framework [39]. As per the previous research, the real-time separation of affected and unaffected Kiwi fruit of ontology security measures and consequences in the Kiwi fruit image is getting a good advantage to detect accurate spot in the Kiwi fruit damages $[23,55]$. From this consideration motivates many of the researchers to make the perfect isolation on the many fruits such as dekopon, densuke watermelon, pineapple, ruby roman grapes, sekai-ichi apple, strawberries arnaud, yubari king melons, and bael or wood apple. All those schemes and processing aspects are not ensuring the spotting of affected and unaffected area fruits. Hence proposed processes are described as the separation of affected and unaffected sekai-ichi apple fruit by using ontology-based Recognition and Isolation Guarantee detection factors $[24,33,42]$. At the same time, the spotting of affected sekai-ichi apple fruit location is somewhat Opposition preserving incompatible factors amongst segmentation and dimension extraction the spotting of unaffected sekai-ichi apple fruits. Hence this research chooses the boosted continuous non-spatial whole attribute extraction (BCNAE) with rhombus based recognition and isolation guarantee spotting (RIGS) scheme separation for computing perfect recognition and isolation in the intelligent agriculture.

\section{Recognition and isolation guarantee spotting (RIGS)}

Recognition and isolation guarantee spotting (RIGS) is a vitally necessary factor to make ontology. Especially, in continuous non-spatial image, the recognition at any target purely depends on the lower level, middle level, and higher-level variables. This recognition features act about proficiency merge-common platforms like the hierarchical model within ontology enabled IoT $[22,56]$. This continuous non-spatial image helps to make better the recognition result of the ontology framework and affected and unaffected sekai-ichi apple fruit separation. Attribute extraction and recognition qualities that are comparable with the existing recognition procedure, the proposed recognition and isolation guarantee spotting (RIGS) have a more prominent attribute retrieved economically [28].

Recognition and isolation guarantee spotting (RIGS) demonstrates to determining the execution of ontology enabled IoT in intelligent agriculture with the lower level characteristics such as dynamic functioning for accuracy and reliability [26, 57]. Moreover, the middle level with the static functioning for generalization. Meanwhile in the upper level for features refining interpretability. These three levels are extracting the attributes in the affected and unaffected sekai-ichi apple fruit separation. Finally, attribute extraction and recognition helps to motivate and qualify the filtering the sekai-ichi apple fruit separation based on the ontology framework and its guarantee spotting (Fig. 2).

Recognition and Isolation Guarantee Spotting (RIGS) are applied to achieving Sekai-ichi apple fruit separation and its processing demands. This process also called the "Rhombus based recognition and Isolation Guarantee Spotting (RIGS)". The guarantee by isolation is very much useful to study the processing consequences from LEVEL 1: image segmentation to affected and unaffected sekai-ichi apple fruit separation for generalizable and interpretable detection of sekai-ichi apple fruit separation and their verification based on the ontology framework updates [58].

\section{Non-spatial image attribute extraction}

The boosted continuous non-spatial whole attribute extraction (BCNAE) is applied to the ontology framework for demonstrating the rhombus based recognition and isolation guarantee spotting (RIGS) for access of Input sekaiichi apple Image Specimen from IS $_{1}$ to IS $\mathrm{I}_{\mathrm{n}}$ for LEVEL ontology formation. This will support to a consequent

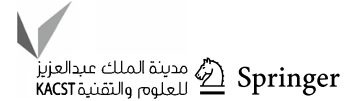


Fig. 2 Recognition and isolation guarantee process flow

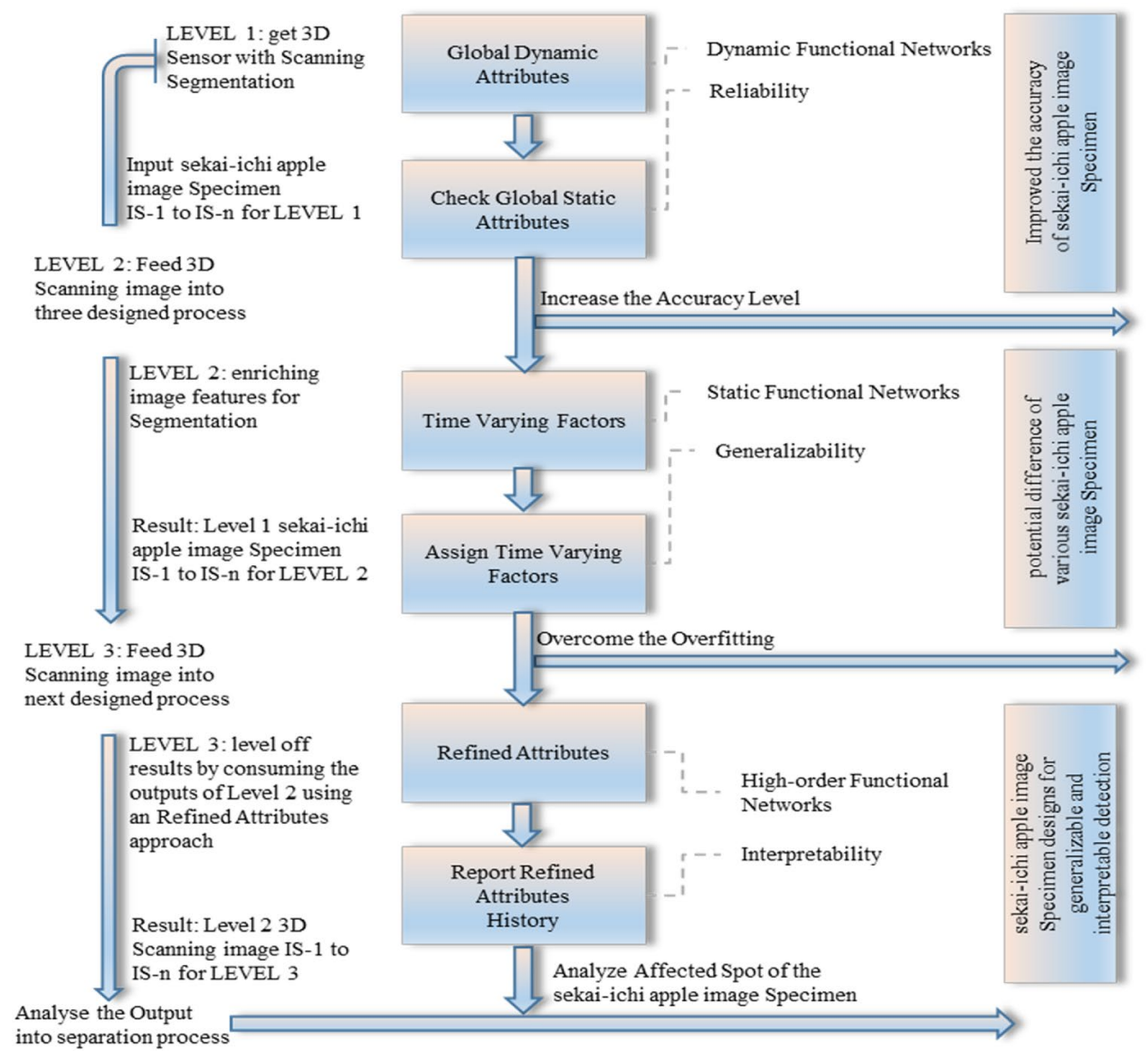

proposed system uses the boosted continuous non-spatial whole attribute extraction (BCNAE) with rhombus based recognition and isolation guarantee spotting (RIGS). RIGS are getting in the input from the 3D sensor will work based on the tetragon based rhombus. The 3D sensor image is creating the Smallest S-vertex tetragon based recognition and isolation guarantee spotting algorithm for the establishment of non-spatial image attribute extraction.

\section{A. "Division A" manual separation affected and unaffected sekai-ichi apple}

Tool Representation 1: shows a semi-automatic sekai-ichi apple separation machine that controls over the mechanical as well as electrical device. This useful to carries separation process by performing semi-automatic manner with assist in the performance of human tasks. A Semi-automatic sekaiichi apple separation machine used to overcoming resistance (affected and unaffected sekai-ichi apple) at many points by applying Non-spatial at any point. This machine motor with four stages; usually propelled by stand between affected and unaffected. 

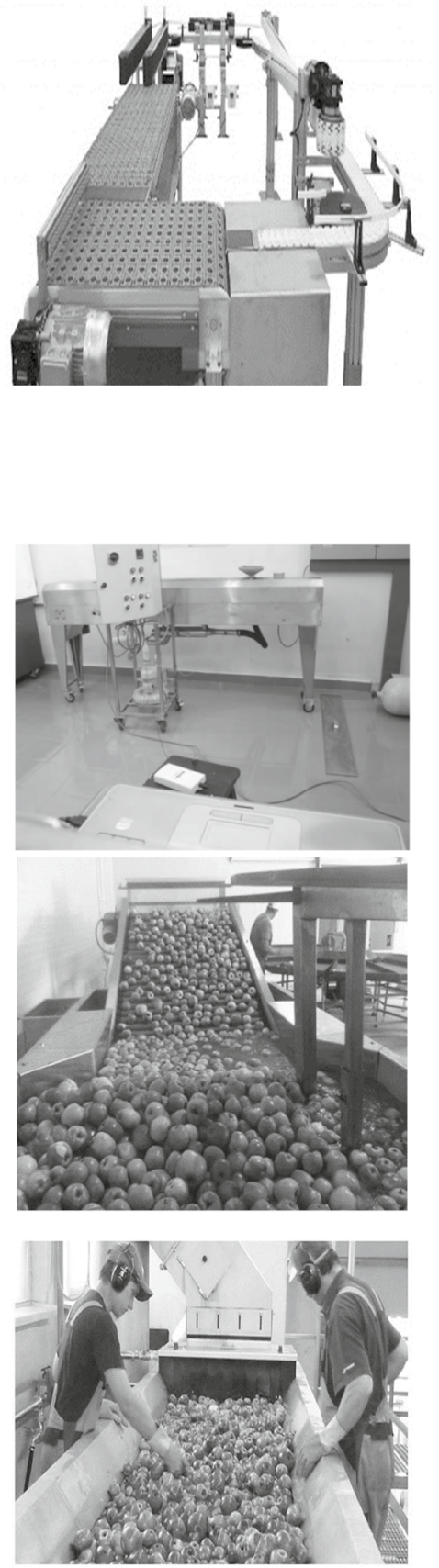

Fig. 3 Division A (Initial Slot with Test Station Accompanying Sekai-Ichi Apple Roller)

Tool Representation 3: shows a semi-automatic sekaiichi apple Separation real time machine that processed with respect to the mechanical and electrical device.

Tool Representation 3: shows a semi-automatic Separation machine with sekai-ichi apple that separate the affected and unaffected sekai-ichi apple at stream flow by applying Non-spatial. This is a first division machine motor with one stage; usually clean and separate unwanted garbage by human tasks.

Tool Representation 4: shows a semi-automatic Separation machine with sekai-ichi apple that separate the affected and unaffected sekai-ichi apple by human tasks.

In the above "Division A" (initial slot with test station accompanying sekai-ichi apple roller), there are a vast number of the sekai-ichi apple is inputted from a manual roller by subjecting to a Non-spatial image attribute extraction process that is employed to the standard "Boosted Continuous Non-spatial whole Attribute Extraction (BCNAE)", which allows for technical help of four tool representations. In this way, the "Division A" process is distinguished between the affected sekai-ichi apple in the initial slot and test station. In such a way, that the sekai-ichi apple may be treated to separate the essential quality of the exact unaffected sekaiichi apple were separated by the "Division A" sekai-ichi apple roller.

\section{B. Semi-automatic “Division B" result image event}

Semi-automatic "Division B" result image event is the process set up with the clarification system, systematic wired cable, two images capturing camera with image capturing chamber. In "Division B" the clarification system manages the initial image checking with the respect the two images capturing camera. The first image capturing camera capture the "Division A" sekai-ichi apple the lower half of the Division A" sekai-ichi apple. This image is stored on the clarification system through systematic wired cable. This will happen until the final sekai-ichi apple received from the roller.

The image capturing camera functions are accompanying the following mode: after getting the input from the roller, the sekai-ichi apples are sent to the camera rotator cabinet. This camera rotator cabinet has always rotated the object for taking the image capturing by using the first image capturing camera. The camera rotator cabinet and image capturing camera took the image of Sekai-ichi apples are on one half. In this stage sekai-ichi, apple is rotated with the help of a camera rotator cabinet. Finally, the sekai-ichi apples rotated with the correct focusing and the snaps to be acquired more than fifty snaps per object.

After getting the image from the front image capturing camera, the Sekai-ichi apples are remains on the camera rotator cabinet. But this camera rotator cabinet is movable and also fixed with rotator belt. This rotator belt always rotates the object for taking another snap by using the second rear image capturing camera. The camera rotator cabinet and image capturing camera took the image of sekai-ichi apples are on another half. In this stage sekai-ichi, apple is rotating with the help of a camera rotator cabinet. Eventually,

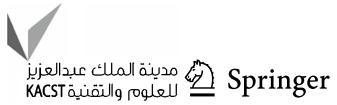


the sekai-ichi apples rotated with the correct focusing and the snaps to be acquired more than fifty snaps per object. The photographs are saved to the clarification system to a prosperous apple that can be separate on the division B stage. Such perspective and predisposition in the sekai-ichi apple image conveyed to the IoT controller having the effect of machinelike separation of each apple. This IoT controller is works based on the intelligent quality sorting system (iQS). This IoT controller decides affected and unaffected individual sekai-ichi apple [60, 61].

From the fifty snaps, in the testbed, five to six snaps are taken to the color frame grabber [20,62]. The IoT controller was applied to adopt an unaffected fruit into the segmentation. This helps to construct the decision making on the image conversion. The image conversion is works based on the sensing image by a rear image sensor [63].

The finalizing system helps to result from image conversion easily, by converts the desired output as per system demands. This is the semi-automatic process because automatic machine drags and drops into the input image on the transition tools. This is very much useful to defeat in large numbers error and increasing the quality of the automatic user input image implementation process.

The out of the Semi-Automatic "Division B" Result Image Event is having the following steps,

Step 1: Reading the input image based on the Image capturing chamber.

Step 2: To match the input image of particular affected sekai-ichi apples image on the "Boosted Continuous Nonspatial whole Attribute Extraction (BCNAE)" based on the post-harvest [55].

Step 3: Identify the ontology framework in the "Boosted Continuous Non-spatial whole Attribute Extraction (BCNAE)". Semi-automatic "Division B" result image event is the most effectual strategy in the focus of making out the procedure to distinguishable healthy separation of sekai-ichi apple.

Semi-automatic "Division B" result image event has to be one more than one working views, especially, image conversion acknowledgment and image matching acknowledgment. These two aspects are used to maintain the image elements between two unique images. This gives the perfect image node identification based on identifier acknowledgment. The following process is establishing the Result Image Conversion process with the direction of segmented image levels. All images can be worked with the Image Conversion, the BCNAE scan the entire object with upper and lower boundary and show the affected spot on the sekai-ichi apple image and number of the spot are determined [27, 42].

\section{C. "Division C" with loT framework for console assembly with charge-coupled device sensing}

IoT framework for console assembly with charge-coupled device sensing scheme requires to demonstrate after a result image conversion, the separation might have made on the affected spot. The IoT framework shows the location of the affected spot and accomplished the exact location of the affected spot. It is also concerning the location by octagon diagonals. Finding a fine-tuned spot is based on the derivation from the vast number region to the smallest region (Figs. 4, 5). This function is extended with each mapping on the octagon diagonals. Finally, the consequence of continuous levels predicts the nearest spot [64].

From the proposed IoT framework for console assembly with charge-coupled device sensing guarantee spotting method is established in the appearing earlier Fig. 6. In discrimination between recognition and isolation is distinct from existing spot identification methods, the first recognition and isolation are treated the Origin Input Image (1) samples, all image comprises the very micro spot on the whole sensed image [44]. In that consideration, this influenced distinguishes are named concerning recognition and isolation. In this era, the origin input image (1) sample image is considered as the octagon diagonals region. Hence, the octagon diagonals sectionalisations are made by using octagon diagonals recognition and isolation spot procedure on the origin input image (1) sample of the input image. This representation denotes a particular image as below specified Eq. 1.

$N=\left\{N_{0}, N_{1}, N_{2} N_{3} N_{4} N_{5} \mid N_{1}\right.$ to $5 \in$ Origin Input Image $\left.{ }_{1 \text { to } 9}\right\}$

In above equation, ' $N$ ' is a large number dissimilar image are arranged purposefully for the input queue with a condition in which everything is regular and unvarying based on the count of Origin Input Image from (1) to (9), this is called the level 1 segmental modification to achieve maximum efficiency in storage capacity, time and cost image. This Optimization is clarifying by giving an example of recognition and isolation in Fig. 2.

To make optimal at level 1, the level 2 optimist and take an isolated view of the image comprises,

$N^{\prime}=\left\{N_{0}^{\prime}, N_{1}^{\prime}, N_{2}^{\prime} N_{3}^{\prime} N_{4}^{\prime} N_{5}^{\prime} \mid N_{1}^{\prime}\right.$ to $5 \in$ Origin Input Image $\left._{1 \text { to } 9}\right\}$

From the above consideration, introduces a level 2 optimist clause two procedures namely of recognition and isolation spot are in equally shares the detection process [24, 58]. Hence, the complete constituting the full quantity of triangle 
Fig. 4 Proposed boosted continuous non-spatial whole attribute extraction (BCNAE) machine architecture

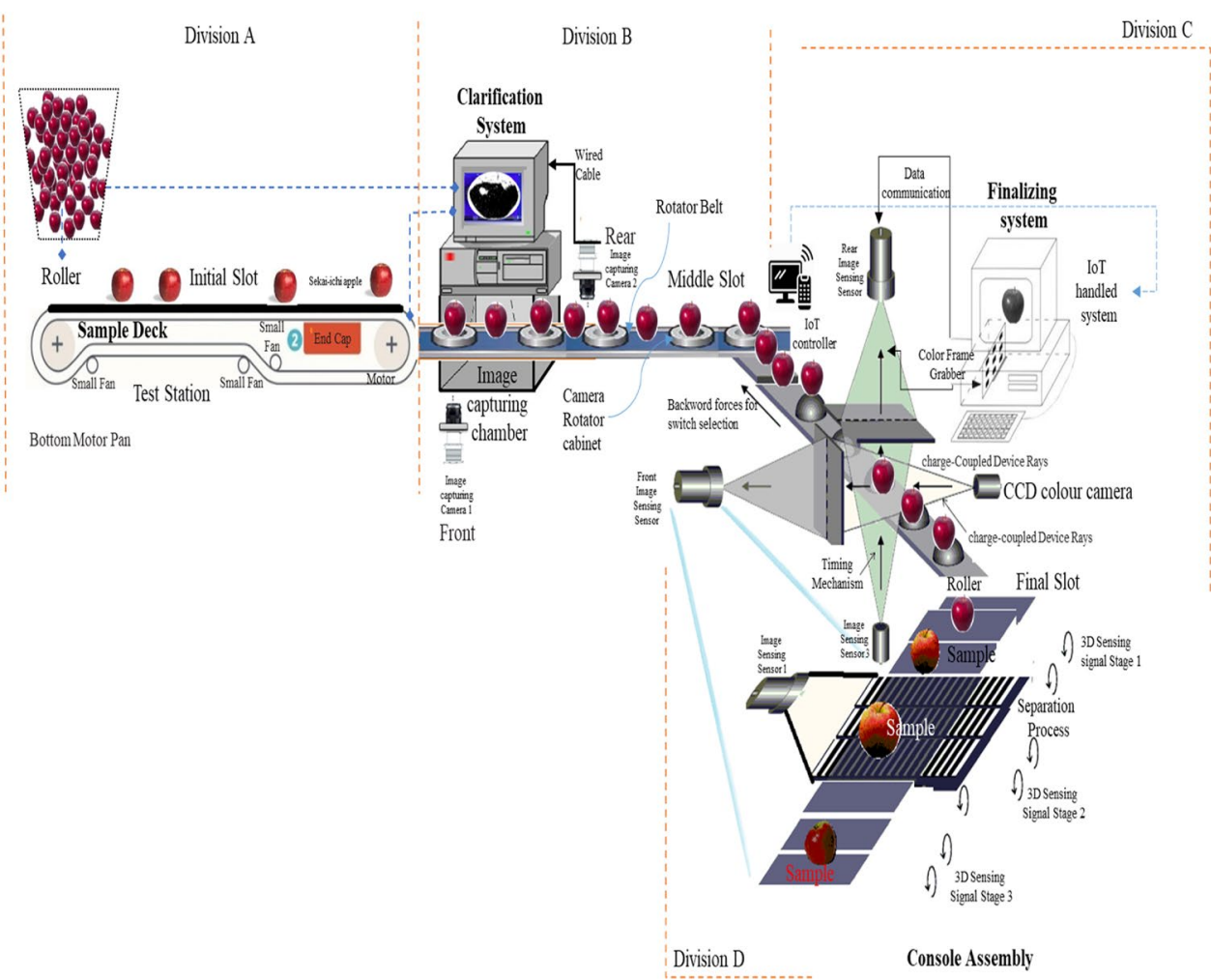

area, the Origin Input Image ' $a$ ' to ' $f$ ' cut in the cardinal number that is the sum of numeral representing of exact location area in which segmentation action at a specific location of S'.

In the level 3, optimize the segmental use best image representation constitutes, diagonals availability. This will have judged by employing the above mathematical statement that two expressions such as $\mathrm{Nt}$ and $\mathrm{Nw}$ in Fig. 6. $N_{c}$ is Manhattan distance if $N_{d}$ is reading the first image discovering procedure. Out of this circumstance, the precise location can be detecting by using

$N^{\prime \prime \prime}=\left\{N_{0}^{\prime \prime \prime}, N_{1}^{\prime \prime \prime}, N_{2}^{\prime \prime \prime}\left|N_{3}^{\prime \prime \prime}\right| N_{4}^{\prime \prime \prime}\left|N_{5}^{\prime \prime \prime}\right| N_{1 \text { to } 5}^{\prime \prime \prime} \in\right.$ Origin Input Image $\left.{ }_{1 \text { to } 9}\right\}$

From the above consideration in Eq. 3, the origin input image ' 1 ' to ' 9 ' separated and expresses the spots used the accurate two location field in which division accomplish at a specific location of $N^{\prime \prime \prime}$.

$\phi_{\text {spot } 1}=\sum_{\text {Lower }=1}^{n}\left[\left(\left|K^{\prime}+K^{\prime \prime}+K^{\prime \prime \prime}\right|^{w}\right)^{\frac{1}{w}}\right]_{x}$

From the above equation, the final optimize segmented optimized image is returned based on The smallest S-vertex octagon based recognition and isolation guarantee spotting algorithm requires the collection of all S-vertex octagon diagonals are based on the below assumption 4 . The exact point located concerning features of the image region of Level 1 Segmented Image(1) to (9) are estimated by make work for a particular affected spot are used as designed above Eq. 4.

To discovery the accurate influenced spot on image region is checked by the most small-scale space of the shortest following Euclidean distance equation,

$\lambda_{\text {precise }}=\sqrt{\sum_{\text {Lower }=n-1}^{9} N_{c}-N_{d} d}=\operatorname{image}(1$ to 9$)$

Consequently, $N_{d}$ is reading the third level the same image of discovering. Hence, the Euclidean distance increases compare to the previous accuracy value. Therefore, level 3 Euclidean distance is compared with the level 2 Euclidean distance, thus the level 3 Euclidean distance of segmented image gets the higher Euclidean distance. Hence optimized segmented image discovering of the affected image is formulated from octagon diagonals region [32].

Taking all input image (1) to input image (9) in the octagon diagonals, the neglecting S-vertex octagon diagonals of the exact spot in following terms 6 . 


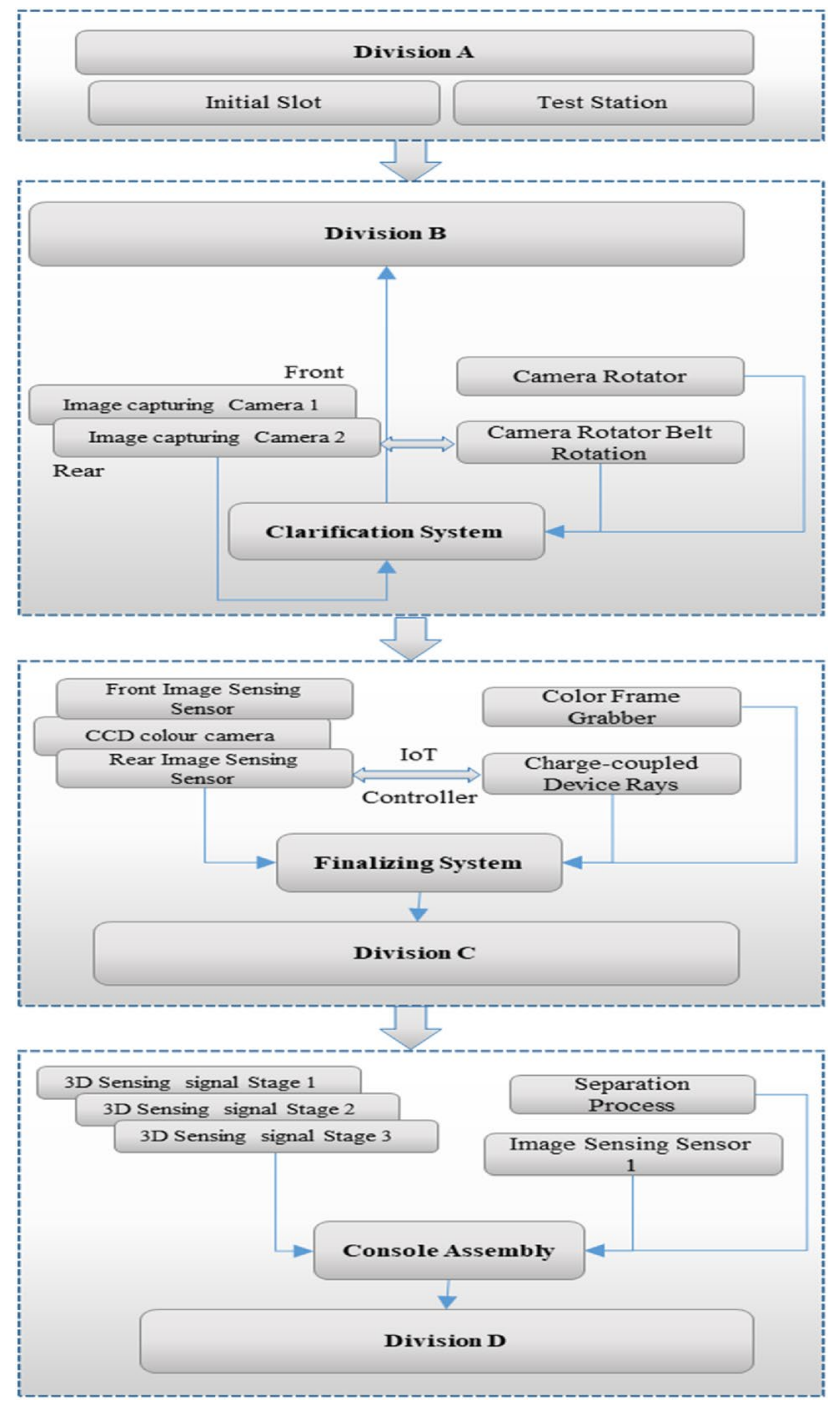

Fig. 5 Semi-automatic "Division B" result image event

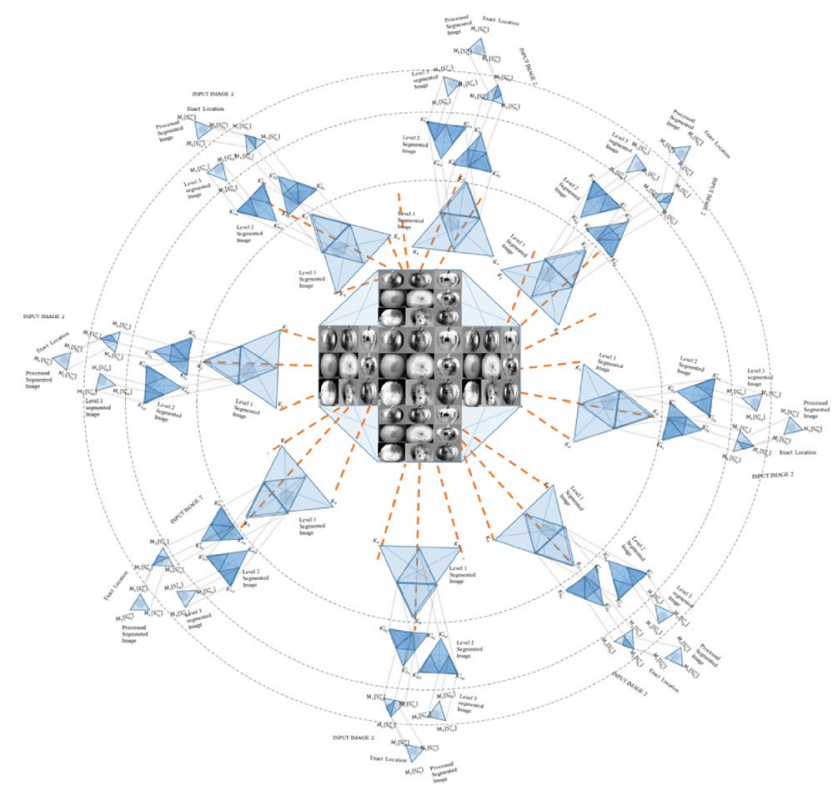

Fig. 6 IoT framework for console assembly with charge-coupled device sensing input images

structure analyzed every input image for the Level 1, Level 2 and level 3 for fine-tuning the separation result. The ontology tree structure obtains by retrieving image; as of information on segmentation with accompanying procedural distinct feature $[28,42,59]$.

\section{D. "Division D" with separation based ontology arrangement of images by ontology tree}

An ontology tree structure with segmentation qualities and performance that are comparable with existent conventional schemes. The ontology tree structure scheme is discussed

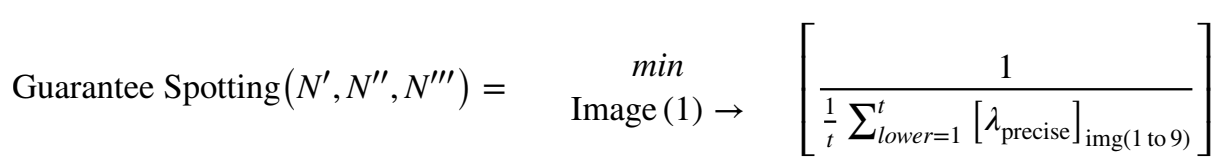

Input Image( 1 to 9$)$

$N^{\prime}, N^{\prime \prime}, N^{\prime \prime \prime}$ is representing to the octagon diagonals region (input image $=1$ to 9 ). Therefore, the exact spot cannot change the precise location. Therefore, S-vertex octagon diagonals characterized by order and way implies the neglecting within the shortest distance of the spot. Therefore, little compensate matching is omitted concerning the Euclidean distance of the segmented image [65].

The recognition and isolation guarantee spotting output were employed to the confirmation analysis based on the "Ontology Tree Structure". The proposed ontology tree with the two categories such as agglomerative method and divisive method. Both the methods are a gain solution from the bottom-up or else top-down manner. Especially the divisive method is the brief process concerning the top-down access, hence automatically the bottom-up process is done on the agglomerative method. This helps to find the spot in terms of accuracy with the help of dimensionality reduction, knowledge discovery, result retrieval, searching and spotting. In this ontology tree structure with segmentation qualities are reported amongst these articles are discussed about the 
lower level, middle level and higher level aggregating with the ontology and segmentation proficiencies [38, 39, 46].

The state of ontologies that a plan is intended to achieve and segmentation terminates behavior intended to achieve detection performance [58]. These ontology tree structure with segmentation schemas are to improve the performance of knowledge discovery, result retrieval, and spotting. The outcomes demonstrate Pointing out ontology concepts with segmentation is predicting and to make better spotting procedure effectively [37, 40].

This Systematic investigation to establish facts of ontology level qualities and performance influence on the spotting with the three levels of processing aspects to make better spotting and allow for a choice between input images (Fig. 7). From the above consideration, the input image is put into ontology level qualities and performance service to make an investigation for a particular spotting or use as designed to Ontology [29].

Here ontology-based spot selection proved for the following condition.

Step 1: Spot the similar image retrieval and spot determination.

Step 2: Build the series of ordered groupings of images within system communities established on the parent node and sub-node

Step 3: Connect the reserve direction of retrieval and spot determination

Step 4: Frame the case-by-case head node on for each one level.

Step 5: Build groupings communities by ontology

Step 6: To establish by deduction, the unavailable spot images into the least right side

Step 7: neglect unavailable spot images compared to retrieval and spot determination.

Step 8: Eventually, ontology level qualities and performance are optimized.
Domain ontology-based ontology level qualities and performance affords the result as the Spotting based Boosted Continuous Non-spatial whole Attribute Extraction (BCNAE) with respect to the above condition [30, 38].

Figure 8 illustrating the level separation based ontology arrangement of images by ontology tree (from the maximum result configuration to minimum result configuration) and optimization-based ontology arrangement of images that act as an ontology tree. If the Ontology tree is disconfirming, the ontology tree can be separated accurately. Hence, that is maximum Level 1 attributes are returned on the Level 1 (lower level). The next Hierarchical level 2 is rationalized for the ontology optimization process [22]. Therefore, that is maximum Level 2 attributes are returned on the Level 2 (middle level). The final hierarchical level 3 ontology optimization proven spot process [56]. Therefore, that is maximum Level 3 attributes are returned on the Level 3 (upper level) [20].

Depending on the degree of relationship between the input image and each level output were rationally depending on the vector space model, it is formed based on.

$\boldsymbol{\mu}_{(a, b)}=\sum_{a=\alpha}^{\beta}[$ image count $(\chi)]+\sum_{b=\eta_{1}}^{\eta_{t}}[$ image count $(\chi)]$

$\eta_{t}$ is the input image its varies from the initial image to the entire input testbed images with spot [20,62]. ' $a$ ' is the set input image count, 'b' is the text spatial location output.

\section{i. Calculate the max separation and min separation of image $(\beta)$}

The forward count image $(\beta)$ and maximum separation as well as minimum separation of image are used to produce state of equilibrium output from $\mu_{(a, b)}$.

Here the minimum separation is calculated based on the below equation.
Fig. 7 Ontology level qualities and performance

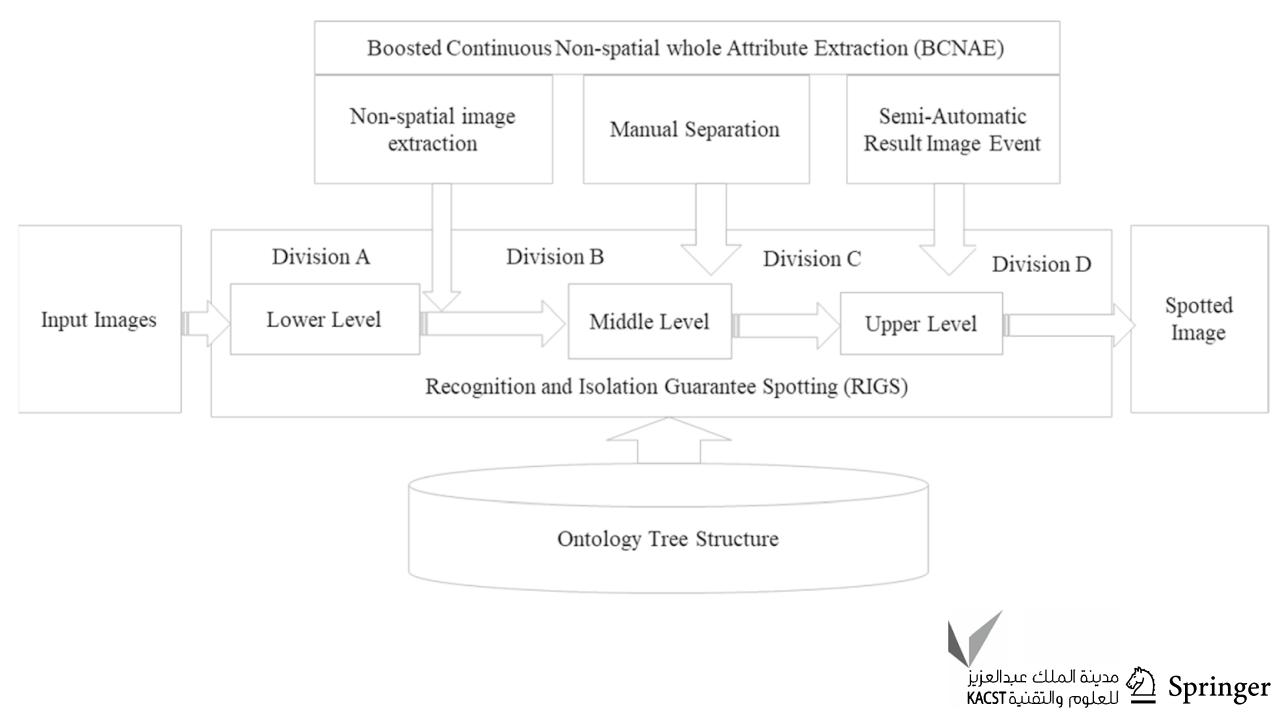


Fig. 8 Separation based ontology arrangement of images by ontology tree

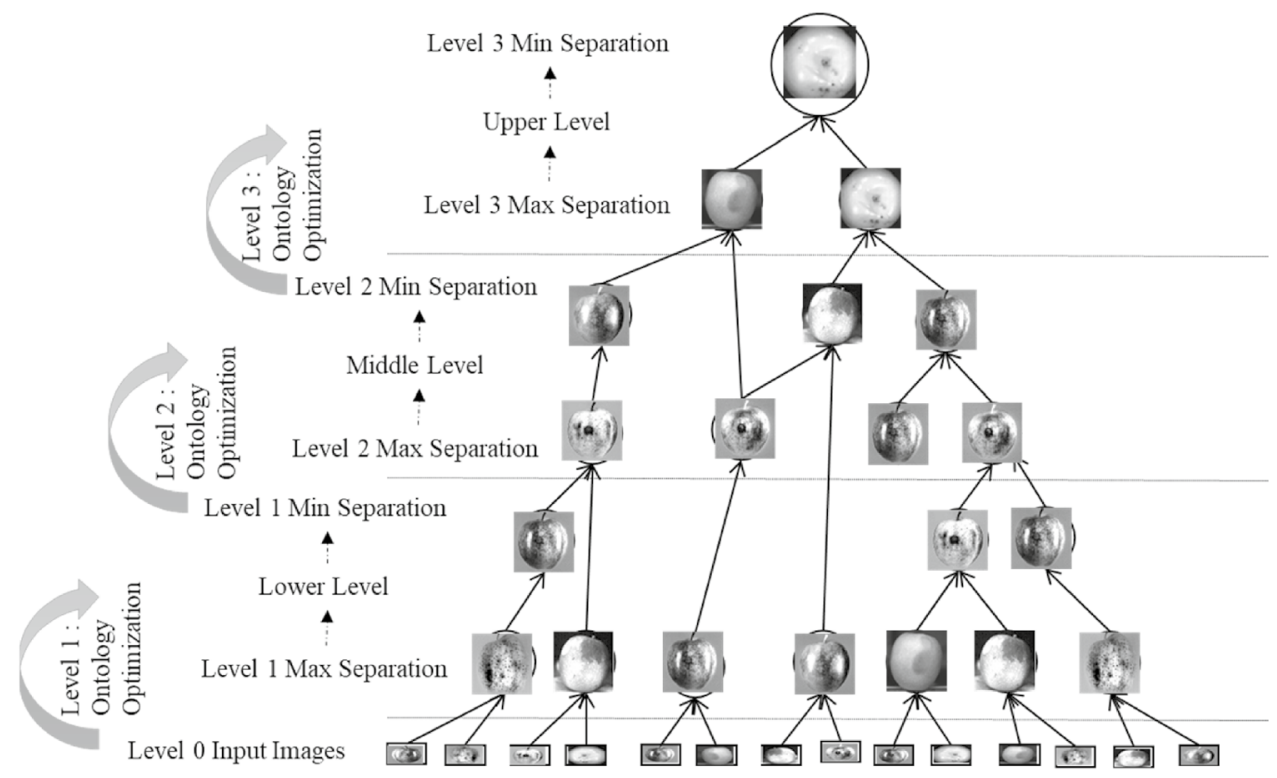

|Min separation|

$=$ Total input image count $* \frac{A v g \_ \text {count }(\text { Image processed })}{\text { Min Spot per time unit }}$

Minimum separation is total input image count are working along with the average count of image were processed with respect to the maximum spot deduction per time unit. i.e.,

|Min separation image|

$=\left(\mu_{(a, b)} * \log \left(\frac{\mathbb{M}[(\text { input image } 1) t o(\text { input image 9)] }}{\text { image count }(\chi)}\right)\right)$

Here the maximum rationalizing is calculated based on the described below equation.

|Max separation|

$$
=\text { Total input image count } * \frac{\text { Max_count }(\text { Image processed })}{\text { Max Possible Spot per time unit }}
$$

Similarly, the maximum rationalizing is total input image count are working along with the maximum count of images were processed with respect to the maximum possible spot deduction per time unit. i.e.,

$$
\begin{aligned}
& \text { |Max separation image } \mid \\
& \qquad=\left(\mu_{(a, b)} * \log \left(\frac{\operatorname{mon}[\text { InputImage } 1]|\ldots| \ldots|\ldots| \ldots \mid \text { Input Image } 9}{\operatorname{image} \operatorname{count}(\chi)}\right)\right)
\end{aligned}
$$

mo is a testbed segmented image count and $\chi$ is an image availability, these two functional aspects are used to produce the maximum separation of image $\beta$. Likewise $\mathbb{M}$ is a test pad segmented image count and $\chi$ is an image availability, these two functional aspects are used to produce the minimum separation of image $\beta$. From the above equation returns the positive result in terms of Min separation of image $(\beta)$. Based on the higher separation value of image spot similarity determination based ontology recognition with ontology formation [39, 46, 62].

\section{Results and discussion}

Extensive result analysis was taken to determine the nature, value, quality, ability, the significance of the services including money and time and labor performance of the system whereby the operator would allocate the automation and manual resources under the optimal separation policy. Figure 9,10,11 and 12 shows the comparison of the results of different artificial machines with and without proposed system processing concerning nature, value, quality, ability, the significance of the services and performance. After balancing the trade-off between the artificial machines and labor performance capabilities, the proposed method is checked with other existing methods in terms of accuracy, time and throughput computation to check its feasibility against proposed separation based ontology arrangement of images by an ontology tree [23, 31, 33]. The result from Figs. 9, 10, 11 and 12 shows that the proposed method is efficient than other existing artificial machines.

Separation is the final step in any post-harvesting system to conclude whether the given image of the sekai-ichi apple is falling affected and unaffected [18, 54]. The photographic image and sensed features are sorted out and broken down into defined classes of affected and unaffected. The affected data from the photographic image used as testing data to 


\section{Min_Accuracy (Affected)}

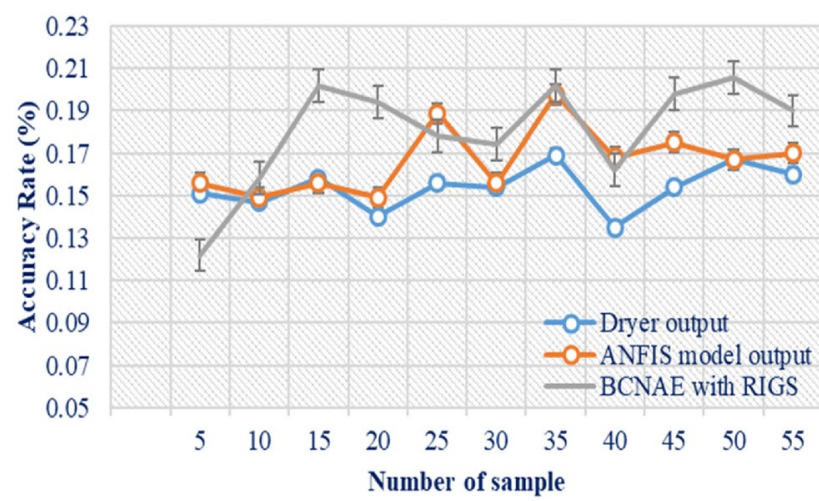

Fig. 9 Minimum accuracy Acc(f)

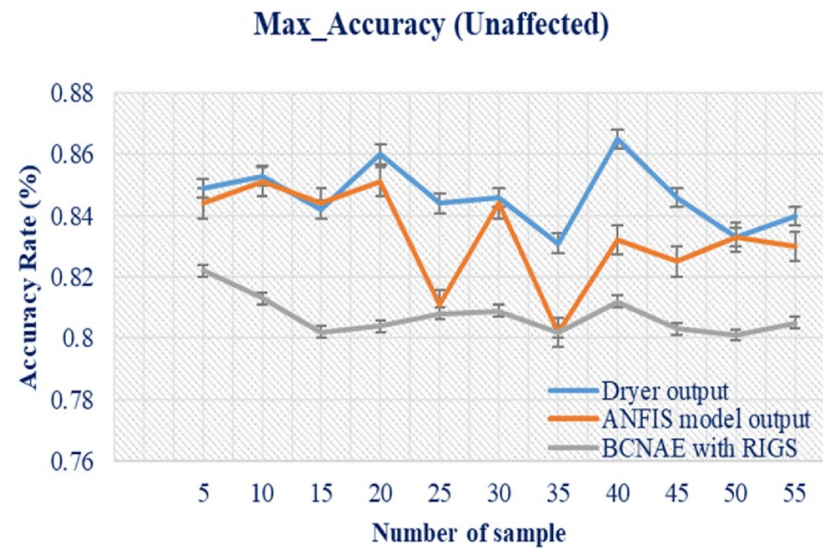

Fig. 10 Maximum accuracy Acc(f)

train the separator. The separation process that can map the inputs image into a particular predefined category is called recognition and isolation guarantee spotting (RIGS), which tends to bring some conclusion from the training data is called the separation model. It can further predict the categories for sensed features.

The separation method which has being nothing more than predicted data, it has the two possible outcomes is called affected and unaffected Sekai-ichi apple separation. Finally, the separator should be evaluated for its accuracy. One of the most crucial consequences in post-harvesting system analysis is the evaluation of the separation results $[18,55]$. Evaluating separation results is the analysis of the output to understand how well it reproduces the original structure of the affected and unaffected sekai-ichi apple image. However, the evaluation of separation results is the most difficult task within the whole roller input sekai-ichi

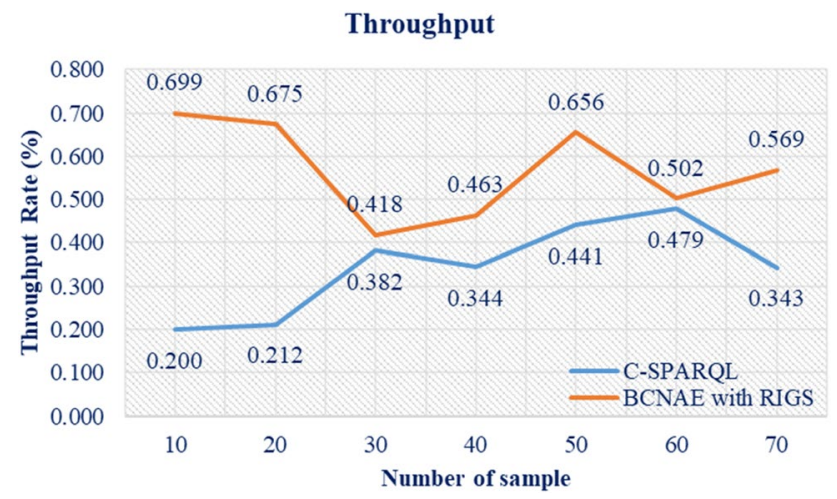

Fig. 11 Throughput comparison of C-SPARQL with proposed BCNAE with RIGS

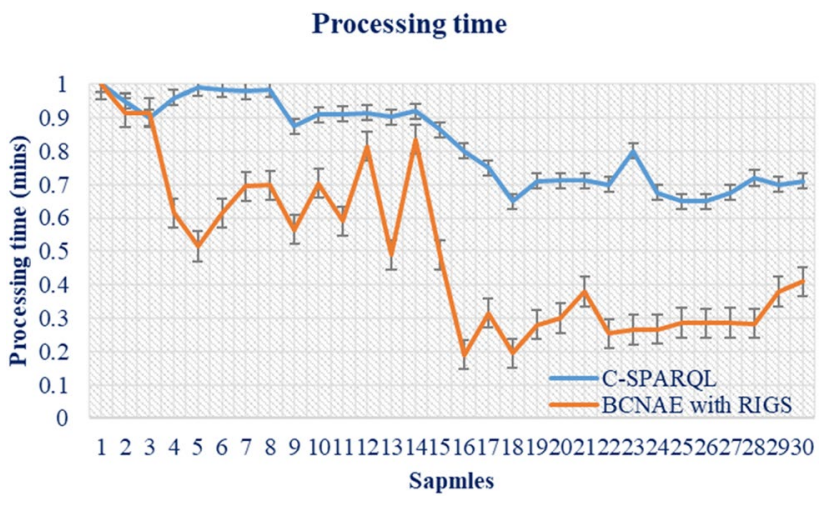

Fig. 12 Processing time comparison of C-SPARQL with BCNAE with RIGS

apple flow. To evaluate the performance of the proposed model following performance metrics are used.

\section{i. Accuracy}

Let $Q_{\mathrm{f}}$ denote the number of input sekai-ichi apple lying in a BCNAE Machine $\left(N^{\prime} N^{\prime \prime} N^{\prime \prime \prime}\right)$ and let $Q \mathrm{j}_{\mathrm{f}}$ be the number of elements of the class Vjin the BCNAE Machine. Then, the maximum Accuracy Acc(f) of the BCNAE Machine $\left(N^{\prime} N^{\prime \prime} N^{\prime \prime \prime}\right)$ with unaffected sekaiichi apple separation is defined as follows:

$[\operatorname{Accuracy}(f)]_{\text {unaffected sekai-ichi }}=\frac{1}{Q_{f}} \max _{j} Q_{j f}$

Accordingly, the overall accuracy of the input, the results is defined as follows

Accuracy $=\sum_{f=1}^{F} \frac{Q_{f}}{q}\left\{[\operatorname{Accuracy}(f)]_{\text {unaffected sekai-ichi }}\right\}$ 
Then, the Minimum Accuracy Acc (f) of the BCNAE Machine $\left(N^{\prime} N^{\prime \prime} N^{\prime \prime \prime}\right)$ with affected sekai-ichi apple separation is defined as follows:

$[\operatorname{Accuracy}(g)]_{\text {affected sekai-ichi }}=\frac{1}{Q_{g}} M I N Q_{i g}$

Accordingly, the overall accuracy of the input, the results is defined as follows

Accuracy $=\sum_{g=1}^{G} \frac{Q_{g}}{q}\left\{[\operatorname{Accuracy}(g)]_{\text {affected sekai-ichi }}\right\}$

Here the $Q_{\mathrm{g}}$ denote the number of input sensed sekai-ichi apple image lying in a BCNAE Machine $\left(N^{\prime} N^{\prime \prime} N^{\prime \prime \prime}\right)$ and let Qig be the number of elements of the class Vjin the BCNAE Machine. Finally, Figs. 9 and 10 displays cumulative accuracy of the existing proportion compare with the proposed system.

The above Fig. 9 illustrates the BCNAE with RIGS upholds its primacy and provides better accuracy performance. In Fig. 9, the mid-half position of the graph, the BCNAE with RIGS achieves $45 \%$ and $150 \%$ better performance compared to Dryer output and ANFIS model output. Standardized execution is sensed while examining the accuracy performance of post-harvest in Boosted Continuous Non-spatial whole Attribute Extraction (BCNAE) apply thoroughly Rhombus based recognition and Isolation Guarantee Spotting (RIGS). Moreover, fraction on the graph shows enhanced accuracy rate of the ANFIS model over the new BCNAE methodology even though the average user manual accuracy drops in BCNAE as compared to the Dryer output.

\section{ii. Throughput}

In the proposed BCNAE method, the less average throughput due to a 3D sensor pattern. The sensor senses the object towards the upper and lower part in a random waypoint and is less likely to reach the limit storage. Therefore, there are more snaps in the machine that leads to fewer inaccuracies. The efficaciousness of the proposed scheme is evaluated on the Non-spatial image attribute extraction, which contains 55 samples that performing different universal separation. After extraction, extracted features like seed, skin, rind, and pomace are normalized and concatenated into the one-dimensional feature vector for each observation [66]. According to the 3D sensor pattern-based semi-automatic separation, the images are divided into observations as labeled and incre- mented in samples of 55 to a maximum. The amount of sekai-ichi apple or other items passing through a BCNAE Machine system is illustrated as the following graph.

The rest of the images is considered as the unlabeled data. From this consideration, the throughput performance evaluation is performed in two levels on a different partition. In the first level, the unaffected object is carried out using a predictive proposed model, build using different separator divisions from A to D. There are four different strong separations used for the first level. In this level proposed BCNAE method is compared with the support continuous queries over RDF data (C-SPARQL) [67]. At the second level, BCNAE is used as a strong separator, and to evaluate the throughput exactness for the best condition that also improves the average recognition rate. From the consideration, the proposed BCNAE method with rhombus based recognition and isolation guarantee spotting (RIGS) performs better than other classifiers in all kind of partition value.

\section{iii. Processing time}

The recognition time of different separators with different stages is illustrated in Fig. 12. Also shows that recognition time increases when the training data size increases with the different nearest count. Evaluation of two-stage semi-automation using different sensors under the 20/80 data partition is shown in the below figure. Experimental results clearly show that in 20/80 partitions, BCNAE with RIGS accuracy is $45 \%$ and $150 \%$ higher than Dryer output and ANFIS model whereas in the $15 / 85$ partitions the above two process processing time is different. Because the proposed scheme has the standardized availability of maximum throughput concerning the number of available unaffected images. In this section, compare the BCNAE with RIGS techniques for both IoT framework and intelligent agricultural actions.

In above Fig. 12, nearly 1000 looping with 70 samples is performed to make a numerical evaluation. For octagonal arrangements of IoT with ontology framework carried out for a different number of images [36, 67]. The observation reveals minimal possible affected object, towards the most occupied areas. Figure 12 underlines the lowest time of the three-level approaches evaluated and visualize any consequence on the throughput enhancement. The collective throughput achievement is spare for BCNAE Machine Architecture provides advancement on the proposed method over conventional technique [37, 67]. 


\section{Conclusion}

In the intelligent agriculture, the post-harvest sector processing schema has directly reduced the losses in an agriculture solid substance separation industry, our research concentrates the sekai-ichi apple fruit quality separation with the lowest degree of wastage in the Post-Harvest process. The BCNAE against the sekai-ichi apple is a safe process at the time of post-harvest. Because, post-harvest losses prevention by precaution deficiencies against quality separation with precise and quick detection of wastage. This proposed system compare with the existing hierarchical model, proposed "Hierarchical Model within Ontology Enabled IoT" for distinguishable healthy separation of sekai-ichi apple. There Hierarchy levels works for dynamic functioning for maintaining the definite number of manual induce with human reliability determination. Although the overfitting and accommodate are also generalizable with the time-varying identification. In addition to this, image segmentation connection process helps to make the proven position of a prominent classification in a particular fruit on the agriculture solid substance. The result against the manual separation and boosted continuous non-spatial whole attribute extraction (BCNAE) machine architecture was compared with the existing techniques. BCNAE are acquiring the higher accuracy with better throughput against the lowest processing time. In the future scope, the proposed system will analyze with the real-time simulation in "big data with $\mathrm{R}$ language" by using the pre-defined data set.

Open Access This article is licensed under a Creative Commons Attribution 4.0 International License, which permits use, sharing, adaptation, distribution and reproduction in any medium or format, as long as you give appropriate credit to the original author(s) and the source, provide a link to the Creative Commons licence, and indicate if changes were made. The images or other third party material in this article are included in the article's Creative Commons licence, unless indicated otherwise in a credit line to the material. If material is not included in the article's Creative Commons licence and your intended use is not permitted by statutory regulation or exceeds the permitted use, you will need to obtain permission directly from the copyright holder. To view a copy of this licence, visit http://creativecommons.org/licenses/by/4.0/.

\section{References}

1. Ahmed K, Shaikh S, Shah AA (2017) Contributions of postharvesting technologies in alleviating poverty: a case study of date palm cluster in Khairpur district, Sindh, Pakistan. In: 2017 IEEE global humanitarian technology conference (GHTC) (pp 1-9), IEEE

2. Alahi MEE, Pereira-Ishak N, Mukhopadhyay SC, Burkitt L (2018) An internet-of-things enabled smart sensing system for nitrate monitoring. IEEE Int Things J 5(6):4409-4417

3. Tseng FH, Cho HH, Wu HT (2019) Applying big data for intelligent agriculture-based crop selection analysis. IEEE Access 7:116965-116974
4. Sanjeevi P, Viswanathan P (2017) NUTS scheduling approach for cloud data centers to optimize energy consumption. Computing 99(12)

5. Sanjeevi P, Viswanathan P (2017) Workload consolidation techniques to optimize energy in cloud: review. Int J Int Protocol Technol 10(2):115-125

6. Sanjeevi P, Viswanathan P (2017) DTCF: deadline task consolidation first for energy minimization in cloud data centers. Int $\mathrm{J}$ Netw Virtual Org Indersci 19(2/3/4):209-233

7. Sanjeevi P, Viswanathan P (2017) Employing smart homes IoT techniques for dynamic provision of cloud benefactors. Int J Crit Comput Based Syst Indersci 7(3)

8. Sanjeevi P, Viswanathan P (2017) A survey on various problems and techniques for optimizing energy efficiency in cloud architecture. Walailak J Sci Technol 14(10)

9. Sanjeevi P, Viswanathan P, Babu MR, Krishna PV (2015) Study and analysis of energy issues in cloud computing. Int $\mathbf{J}$ Appl Eng Res 10(7):16961-16969

10. Sanjeevi P, Balamurugan G, Viswanathan P (2017) The improved DROP security based on hard ai problem in cloud. Int J Int Protocol Technol 9(4):207-217

11. Sanjeevi P, Viswanathan P (2015) A green energy optimized scheduling algorithm for cloud data centers. In: IEEE international conference on computing and network communications, Trivandrum, pp 941-945

12. Sanjeevi P, Viswanathan P (2016) Towards energy-aware job consolidation scheduling in cloud. In: International conference on inventive computation technologies (ICICT 2016), IEEE Xplore, pp 361-366

13. Kesavan G, Sanjeevi P, Viswanathan P (2016) A 24 hour IoT framework for monitoring and managing home automation. In: International conference on inventive computation technologies (ICICT 2016), IEEE Xplore, pp 367-371

14. Sanjeevi P, Ashwin M, Manikandan R, Karthick Raghunath KM, Anantha Raman GR (2016) Heterogeneous internet of things organization predictive analysis platform for apple leaf diseases recognition,computer communications, Volume 154, 2020, pp 99-110

15. Ramasamy M, Santhanam P, Muniyappan A, Lakshmanan SK, Pandiyan S (2020) A novel methodology for the development of an optimal agricultural crop field using Internet of Things. Comput Intell pp 1-16. https://doi.org/10.1111/coin.12308

16. Sanjeevi P, Prasanna S, Siva Kumar B, Gunasekaran G, Alagiri I, Vijay Anand R (2020) Precision agriculture and farming using Internet of Things based on wireless sensor network. Trans Emerging Tel Tech e3978. https://doi.org/10.1002/ett.3978

17. Mehrotra P, Mehrotra A, Suryawanshi R (2009) Effect of post harvest deterioration on efficiency of clarification and crystallization process of white sugar manufacture of $\operatorname{CoS} 91269$ cane variety. In: 2009 Second international conference on emerging trends in engineering \& technology (pp 781-784), IEEE

18. Rominger C, Emert S, Ushimaru K (2012) Development of a sustainable food supply chain by post harvest program-an approach to a sustainable solution to food delivery and waste problems. In: 2012 IEEE global humanitarian technology conference (pp 230-236), IEEE

19. Chaisu K (2018) Biological control of post-harvest banana diseases using antagonistic bacteria in Thailand. In: 2018 IEEE 5th international conference on engineering technologies and applied sciences (ICETAS) (pp 1-4), IEEE

20. Agarwal R, Fernandez DG, Elsaleh T, Gyrard A, Lanza J, Sanchez L, Issarny V (2016) Unified IoT ontology to enable interoperability and federation of testbeds. In: 2016 IEEE 3rd World Forum on Internet of Things (WF-IoT) (pp 70-75), IEEE 
21. Jin Y, Li G, Zhang H (2018) Evaluation of regional rural information environment based on fuzzy method in the Era of the internet of things. IEEE Access 6:78530-78541

22. Abbasi KM, Khan TA, Haq IU (2019) Hierarchical modeling of complex internet of things systems using conceptual modeling approaches. IEEE Access 7:102772-102791

23. Arruda MF, Bulcão-Neto RF (2019) Toward a lightweight ontology for privacy protection in IoT. In: Proceedings of the 34th ACM/SIGAPP symposium on applied computing (pp 880-888), ACM.

24. Chen WL, Lin YB, Ng FL, Liu CY, Lin YW (2019) Rice talk: rice blast detection using internet of things and artificial intelligence technologies. IEEE IoT J

25. Horng GJ, Liu MX, Chen CC (2019) The smart image recognition mechanism for crop harvesting system in intelligent agriculture. IEEE Sens J

26. Li H, Liu S, Duan Q, Li W (2018) Application of Multi-Sensor Image Fusion of Internet of Things in Image Processing. IEEE Access 6:50776-50787

27. Liu S, Guo L, Webb H, Ya X, Chang X (2019) Internet of things monitoring system of modern eco-agriculture based on cloud computing. IEEE Access 7:37050-37058

28. Min Z, Bei W, Chunyuan G (2011) Application study of precision agriculture based on ontology in the internet of things environment. In: International conference on applied informatics and communication (pp 374-380), Springer, Berlin

29. Beck H, Morgan K, Jung Y, Grunwald S, Kwon HY, Wu J (2010) Ontology-based simulation in agricultural systems modeling. Agric Syst 103(7):463-477

30. Bernstein A, Provost F, Hill S (2005) Toward intelligent assistance for a data mining process: an ontology-based approach for cost-sensitive classification. IEEE Trans Knowl Data Eng 17(4):503-518

31. Alsubaei F, Abuhussein A, Shiva S (2019) Ontology-based security recommendation for the internet of medical things. IEEE Access 7:48948-48960

32. Sai Z, Fan Y, Yuliang T, Lei X, Yifong Z (2016) Optimized algorithm of sensor node deployment for intelligent agricultural monitoring. Comput Electron Agric 127:76-86

33. Bali A, Al-Osta M, Abdelouahed G (2017) An ontology-based approach for IoT data processing using semantic rules. In: International SDL forum (pp 61-79), Springer, Cham

34. Gupta M, Singh R (2006) Experience management framework for managing innovation in post-harvest resource management. In: 2006 IEEE international conference on management of innovation and technology (Vol 1, pp 375-379), IEEE

35. Khalid NS, Abdullah AH, Shukor SAA, ASFS, Mansor H, Dalila NDN (2017) Non-destructive technique based on specific gravity for post-harvest mangifera indica L. cultivar maturity. In: 2017 Asia Modelling Symposium (AMS) (pp 113-117), IEEE

36. De Clercq PA, Hasman A, Blom JA, Korsten HH (2001) The application of ontologies and problem-solving methods for the development of shareable guidelines. Artif Intell Med 22(1):1-22

37. El Bouhdidi J, Ghailani M, Fennan A (2012) Towards an optimized design of individualized learning paths: an approach based on ontology and multi-agents system. Int J Comput Sci Issues (IJCSI) 9(6):362

38. Isaza G, Castillo A, López M, Castillo L (2009) Towards ontology-based intelligent model for intrusion detection and prevention. In: Computational intelligence in security for information systems (pp 109-116), Springer, Berlin

39. Liu XY, Li XB, Fu XD, Zhang JC (2014) Research on PPHIIS based on ontology model. In: Applied mechanics and materials (Vol 519, pp 66-69), Trans Tech Publications

40. Gomez-Perez A, Benjamins VR (1999) Applications of ontologies and problem-solving methods. AI Magazine 20(1):119-119
41. Lin H, Yifen L, Chen Y, Xiangjia K, Junian Z (2011) Technologies of post-harvest handling and storage for longan fruits. In: 2011 International conference on new technology of agricultural (pp. 758-763), IEEE

42. Maleki E, Belkadi F, Boli N, Van Der Zwaag BJ, Alexopoulos K, Koukas S, Mourtzis D (2018) Ontology-based framework enabling smart product-service systems: application of sensing systems for machine health monitoring. IEEE Int Things J 5(6):4496-4505

43. Kianoush S, Raja M, Savazzi S, Sigg S (2018) A cloud-IoT platform for passive radio sensing: challenges and application case studies. IEEE Int Things J 5(5):3624-3636

44. Lan L, Shi R, Wang B, Zhang L (2019) An IoT unified access platform for heterogeneity sensing devices based on edge computing. IEEE Access 7:44199-44211

45. Geng X, Zhang Q, Wei Q, Zhang T, Cai Y, Liang Y, Sun X (2019) A mobile greenhouse environment monitoring system based on the internet of things. IEEE Access 7:135832-135844

46. Kumar V (2015) Ontology based public healthcare system in internet of things (IoT). Procedia Comput Sci 50:99-102

47. Kamilaris A, Gao F, Prenafeta-Boldú FX, Ali MI (2016) Agri-IoT: a semantic framework for internet of things-enabled smart farming applications. In: 2016 IEEE 3rd world forum on internet of things (WF-IoT) (pp 442-447), IEEE

48. Xiao G, Guo J, Da Xu L, Gong Z (2014) User interoperability with heterogeneous IoT devices through transformation. IEEE Trans Industr Inf 10(2):1486-1496

49. Lutfy OF, Selamat H, Noor SM (2015) Modelling of a conveyorbelt grain dryer utilizing a sigmoid network. In: 2015 10th Asian control conference (ASCC) (pp 1-5). IEEE

50. Lu CH, Tsai CE (2019) IoT-enabled cross-field and reconfigurable service provisioning with user-centered design. IEEE Syst J

51. Hamidi H, Fazeli K (2018) Using internet of things and biosensors technology for health applications. IET Wireless Sens Syst 8(6):260-267

52. Guzman-Miranda H, Nápoles J, Patiño A, Mateos R, Matías M, Amador J, Pérez-Córdoba J (2009) Realization of a flexible platform for fruit inspection and classification applications with emphasis in rapid prototyping and development. In: 2009 35th annual conference of IEEE industrial electronics (pp 2874-2879), IEEE

53. Oluwo A, Khan MR, Salami MJE (2015) Intelligent temperature control of a tropical post-harvest storage system. In: 2015 10th Asian Control Conference (ASCC) (pp 1-6). IEEE

54. Purandare H, Ketkar N, Pansare S, Padhye P, Ghotkar A (2016) Analysis of post-harvest losses: an internet of things and machine learning approach. In: 2016 International conference on automatic control and dynamic optimization techniques (ICACDOT) (pp 222-226), IEEE

55. Yifan Z, Honghui R (2011) Effects of high voltage electrostatic field treatment on post-harvest physiology of Kiwifruit. In: 2011 International conference on new technology of agricultural (pp 994-997), IEEE

56. Yang Z, Ding Y, Jin Y, Hao K (2018) Immune-endocrine system inspired hierarchical coevolutionary multiobjective optimization algorithm for IoT service. In: IEEE transactions on cybernetics

57. Perera C, Zaslavsky A, Liu CH, Compton M, Christen P, Georgakopoulos D (2013) Sensor search techniques for sensing as a service architecture for the internet of things. IEEE Sens J 14(2):406-420

58. Zheng R, Wang H, Zhao J (2019) A unified management framework for EIoT systems based on metadata and event detection. IEEE Access 7:112629-112638

59. Xu G, Cao Y, Ren Y, Li X, Feng Z (2017) Network security situation awareness based on semantic ontology and user-defined rules for Internet of Things. IEEE Access 5:21046-21056 
60. Wan J, Lai CF, Song H, Imran M, Jia D (2019) Software-defined industrial internet of things. Wireless communications and mobile computing, 2019

61. Westerlund M, Leminen S, Rajahonka M (2014) Designing business models for the internet of things. Technol Innovat Manag Rev $5-14$

62. Lanza J, Sanchez L, Santana JR, Agarwal R, Kefalakis N, Grace P, Cirillo F (2018) Experimentation as a service over semantically interoperable Internet of Things testbeds. IEEE Access 6:51607-51625

63. Xu C, Yang HH, Wang X, Quek TQ (2019) Optimizing information freshness in computing enabled IoT networks. IEEE Int Things J

64. Yang Z, Jin Y, Hao K (2018) A bio-inspired self-learning coevolutionary dynamic multiobjective optimization algorithm for internet of things services. In: IEEE transactions on evolutionary computation
65. Sattar H, Bajwa IS, Amin RU, Sarwar N, Jamil N, Malik MA, Shafi U (2019) An IoT-based intelligent wound monitoring system. IEEE Access 7:144500-144515

66. Niu K, Zhao X, Li F, Li N, Peng X, Chen W (2019) UTSP: userbased two-step recommendation with popularity normalization towards diversity and novelty. IEEE Access 7:145426-145434

67. Barbieri DF, Braga D, Ceri S, Valle ED, Grossniklaus M (2009) C-SPARQL: SPARQL for continuous querying. In: Te 18th international conference on World wide web-WWW'09 (pp 1061-1062)

Publisher's Note Springer Nature remains neutral with regard to jurisdictional claims in published maps and institutional affiliations. 